\title{
Thoracoscopic Transmitral Myectomy of Apical HOCM with Concomitant Valve Procedure with 3D Printing Technique Assistant: Response to Letter to the Editor
}

\author{
ASHOK KUMAR C J1 \\ ${ }^{1}$ The University of Mississippi Medical Center
}

April 26, 2021

\begin{abstract}
We thank Shixiong Wei et al for their interest in our technical paper on Robotic Septal myectomy for HOCM and their letter to the editor. Their technique of using the Computed Tomography Angiography (CTA) along with 3D Printing technology for the better delineation of the anatomy of HOCM has been an evolving way to approach the problem. As they have mentioned in their letter, we used cardiac magnetic resonance imaging (MRI) predominantly for all our patients who undergo septal myectomy. Using 3D printing technology augmenting CTA or MRI can certainly augment the surgical planning in complex patients. In their case report, Wei et al have shaved off most of the subvalvular tissue along with the mitral valve. Removal of the entire mitral valve definitely would have enhanced the exposure to the mid-ventricular cavity and the apex for their thoracoscopic approach. It will be interesting to know whether they tried to repair the valve before replacement. Our technique of Robotic mitral valve repair, septal myectomy, and particularly papillary muscle re-orientation tries to preserve the sub-valvular apparatus even after completely relieving the mid-cavitary obstruction and this may provide better ventricular re-modeling. We congratulate them on providing a successful surgical option to this complex subset of patients using novel evolving technology and meticulous surgical planning.
\end{abstract}

\section{Hosted file}

Response to Letter to the Editor from Wei et al.pdf available at https://authorea.com/ users/410100/articles/519593-thoracoscopic-transmitral-myectomy-of-apical-hocm-withconcomitant-valve-procedure-with-3d-printing-technique-assistant-response-to-letter-tothe-editor 